Federal Reserve Bank of Minneapolis

Research Department Working Paper

A GENERALIZED VARIANCE BOUNDS TEST

Tryphon Kollintzas*

Working Paper 352

June 1987

NOT EOR DISTRIBUTION

WITHOUT AUTHOR'S APPROVAL

ABSTRACT

This paper derives a variance bounds test for a broad class of linear rational expectations models. According to this test if observed data accords with the model, then a weighted sum of autocovariances of the covariance-stationary components of the endogenous state variables should be nonnegative. The new test reinterprets its forefather--West's [1986] variance bounds test-and extends its applicability by not requiring exogenous state variables in order to be tested. The possibility of the test's application to nonlinear models is also discussed.

*Federal Reserve Bank of Minneapolis and University of Pittsburgh. I have benefited from comments by Lawrence Christiano, Edward Green, Hugo Hopenhayn, Patrick Kehoe, Thomas Sargent, Kenneth West, and the stimulating environment of the Federal Reserve Bank of Minneapolis. The financial support of the $\mathrm{Na}-$ tional Science Foundation is gratefully acknowledged.

The views expressed herein are those of the author and not necessarily those of the Federal Reserve Bank of Minneapolis or the Federal Reserve System. The material contained is of a preliminary nature, is circulated to stimulate discussion, and is not to be quoted without permission of the author. 
1. Introduction

In a recent issue of this journal West [1986] tested a linear rational expectations (LRE) version of the production smoothing-buffer stock model of inventories. He hypothesized that the covariance-stationary components of production, sales, and inventories are consistent with the optimal policy of the underlying dynamic maximization problem. Then, he showed that the unconditional expectation of the difference between the value of the objective function under the optimal policy and the value of the objective function under a policy where inventories are identically zero, is equal to a weighted sum of variances and covariances of production, sales, and inventories. Thus, he proceeded to test whether the nonnegativity of this sum was satisfied by the covariance-stationary components of the observed data.

The purpose of this paper is to reinterpret and generalize West's condition for a broad class of LRE models. It is shown that the unconditional expectation of the difference between the value of the objective function of the underlying dynamic problem under an optimal policy and the value of this function under any feasible policy, such that the difference between the corresponding state paths is a covariance-stationary process, is a weighted sum of autocovariances of that process. Thus, this sum must be nonnegative for any covariance-stationary difference between an optimal state path and a feasible state path. There are two standout implications of this result. First, if observed data accord with the optimal policy (i.e., the theory) then their covariance-stationary components should satisfy the above condi- 
tion. For, as shown below, the nonstationary components of the endogenous state variables qualify as the state path associated with a feasible policy. This is the reinterpretation result. Second, unlike West's condition, the one derived here does not require exogenous state variables in order to be tested. This is the generalization result and is a consequence of the fact that the condition derived here exploits some of the other necessary conditions while West's does not.

Section 2 sets up a general LRE model and reviews the standard necessary conditions for its solution. Section 3 proves the necessity of the new condition and discusses its extension to nonlinear rational expectations models. Section 4 presents two examples. The second example involves West's inventory model and juxtaposes the new condition to his.

\section{A General LRE Model}

Let $\{\xi(t): t \in \mathbf{N}\}, N=\{0, \pm 1, \ldots\}$, be a stochastic process on a probability space $(\underline{0}, \Omega, P)$, where $\xi(t)$ is an $\mathrm{n}_{\xi} \times 1$ dimensional vector of exogenous state variables at the beginning of period $t$. Also, let $\Omega_{t}$ be the $\sigma$-algebra generated by the sequence of random variables $(\ldots, \xi(t-1), \xi(t)), t \varepsilon N . \Omega_{t}$ represents the information available to the system at the beginning of period t. Clearly, $\Omega_{t} \subset \Omega_{t+1} \subset \Omega, \forall t \in N$. $E(\cdot)$ denotes the unconditional expectations operator with respect to $P$. That is, for any integrable function (•) with respect to $P, E(\cdot)=\int(\cdot) d P=$ $\int(\cdot) P(d \omega) . \quad E_{t}(\cdot)$ denotes the conditional expectations operator with respect to $P$, given $\Omega_{t}$. That is, for any integrable function (•) with respect to $P$ such that $E(\cdot)<\infty, \int_{A}(\cdot) P(d \omega)=$ 
$\int_{A} E_{t}(\cdot) P(d \omega), \quad \forall A \quad \varepsilon \Omega_{t} . \quad\{\xi(t): t \varepsilon\{\tau, \tau+1, \ldots\}, \tau \in N\}$ takes values in $f_{\tau}^{\xi}$. That is, the spaces of sequences $\xi=(\xi(\tau), \xi(\tau+1), \ldots)$, $\tilde{\xi}=(\tilde{\xi}(\tau), \tilde{\xi}(\tau+1), \ldots)$, etc., such that

$$
\sum_{t=\tau}^{\infty} B^{t-\tau} E \xi(t) \cdot \tilde{\xi}(t)<\infty,
$$

where $\beta \varepsilon(0,1)$ is the discount factor in all periods. Also, let $x(t)$ be an $n_{x} \times 1$ dimensional vector of endogenous state variables at the beginning of period $t$. Then, a variety of LRE models can be stated as a problem of the form:

$$
\max _{\{u(t)\}_{t=\tau}^{\infty}} \lim _{T \rightarrow \infty} E \sum_{\tau} \sum_{t=\tau}^{T} \beta^{t-\tau} f[\xi(t), x(t), u(t)]
$$

subject to:

$$
\begin{aligned}
& u(t) \Omega_{t} \text {-measurable } \\
& x(t+1)=g[\xi(t), x(t), u(t)] \\
& x(\tau)=\bar{x} \text { (given) } \\
& \{x(t)\}_{t=\tau}^{\infty} \varepsilon f_{\tau}^{x}
\end{aligned}
$$

where $u(t)$ is an $n_{u} \times 1$ dimensional vector of control variables in period $t$,

$$
\left.f\right|^{t}=\left[\begin{array}{l}
\phi_{\xi} \\
\phi_{x} \\
\phi_{u}
\end{array}\right] \cdot\left[\begin{array}{l}
\xi(t) \\
x(t) \\
u(t)
\end{array}\right]+\frac{1}{2}\left[\begin{array}{l}
\xi(t) \\
x(t) \\
u(t)
\end{array}\right] \cdot\left[\begin{array}{lll}
\phi_{\xi \xi} & \phi_{\xi x} & \phi_{\xi u} \\
\phi_{X \xi} & \phi_{x x} & \phi_{x u} \\
\phi_{u \xi} & \phi_{u x} & \phi_{u u}
\end{array}\right]\left[\begin{array}{l}
\xi(t) \\
x(t) \\
u(t)
\end{array}\right],
$$

$\dot{\phi}_{i}$ and $\phi_{i j}$ are appropriately dimensioned vectors and matrices, respectively, such that 
(6a)

$$
\phi_{i j}{ }^{\prime}=\phi_{j i}
$$

$$
\left.g\right|^{t}=r_{x \xi^{\xi}}(t)+r_{x x} x(t)+r_{x u} u(t),
$$

$\gamma_{x i}$ are appropriately dimensioned matrices, such that there exists an $n_{u} \times n_{x}$ dimensional matrix $\delta_{u x}$ with the property

$$
\delta_{u x_{x u}^{Y}}=I
$$

and $f_{\tau}^{x}$ is the space of sequences $x=(x(\tau), x(\tau+1), \ldots), \tilde{x}=$ $(\tilde{x}(\tau), \tilde{x}(\tau+1), \ldots)$, etc., such that

$$
\sum_{t=\tau}^{\infty} \beta^{t-\tau} \operatorname{Ex}(t)+\tilde{x}(+)<\infty .
$$

It follows from the preceding formulation that $x(t+1)$ is $\Omega_{t}$-measurable for all $t \varepsilon\{\tau, \tau+1, \ldots\}$. Thus, decisions at time $t$ depend only on the past history of the $\{\xi(t) ; t \in \mathbf{N}\}$ process and $\bar{x}$.

It should be noted that no a priori curvature restrictions have been imposed on $\left.f\right|^{t}$. Also, no explicit law of motion for the $\{\xi(t): t \varepsilon N\}$ process has been postulated. On the other hand, $\{x(t)\}_{t=\tau}^{\infty} \in f_{\tau}^{X}$ can be relaxed. For our purposes it suffices that $\beta^{(T-\tau)}\left|E_{\tau} x(T)^{\prime} x(T)\right| \rightarrow 0$ as $T \rightarrow \infty, \forall \bar{x} \in R^{n x}$. Moreover, (7a) can be altogether eliminated. Its implication is, of course, that there are $n_{x}$ controls at the most in the system. Effectively, this excludes all these models whose solution cannot be characterized by Euler conditions. Now, it will be convenient to transform $P$ as follows. 
$-5-$

Fact 1: Given (6a) and (7a), (P) is equivalent to:

(1')

$$
\max _{\{x(t+1)\}_{t=\tau}^{\infty} \lim _{T \rightarrow \infty} E_{\tau} \sum_{t=\tau}^{T} \beta^{t-\tau} h[\xi(t), x(t), v(t)]}
$$

subject to:

(2') $\quad x(t+1) \Omega_{t}$-measurable

(3') $\quad v(t)=x(t+1)-x(t)$

( $\left.P^{\prime}\right)$

(4') $\quad x(\tau)=\bar{x}$

(5') $\quad\{x(t)\}_{t=\tau}^{\infty} \in f_{\tau}^{x}$.

where:

$\left(6^{\prime}\right)$

$$
\left.h\right|^{t}=\left[\begin{array}{l}
k \\
l \\
m
\end{array}\right]+\left[\begin{array}{l}
\xi(t) \\
x(t) \\
v(t)
\end{array}\right]+\frac{1}{2}\left[\begin{array}{l}
\xi(t) \\
X(t) \\
v(t)
\end{array}\right]+\left[\begin{array}{lll}
N & 0 & P \\
O^{\prime} & Q & R \\
P^{\prime} & R & S
\end{array}\right]\left[\begin{array}{l}
\xi(t) \\
x(t) \\
v(t)
\end{array}\right]
$$

(6a') $\quad N^{\prime}=N, Q^{\prime}=Q$, and $S^{\prime}=S$

$$
\begin{aligned}
& \ell=\phi_{x}+\left(I-\gamma_{x x}\right)^{\prime} \delta_{u x}{ }^{\prime} \phi_{u} \\
& m=\delta_{u x}{ }^{\prime} \phi_{u} \\
& 0=\phi_{\xi x}-\gamma_{x \xi}{ }^{\prime} \delta_{u x}{ }^{\prime}+\phi_{\xi x} \delta_{u x}\left(I-\gamma_{x x}\right)-\gamma_{x \xi}{ }^{\prime} \delta_{u x}{ }^{\prime} \phi_{u u} \delta_{u x}\left(I-\gamma_{x x}\right) \\
& D=\phi_{\xi u} \delta_{u x}-\gamma_{x \xi}{ }^{\prime} \delta_{u x} \phi_{u u} \delta_{u x} \\
& Q=\phi_{x x}+\left(I-\gamma_{x x}\right)^{\prime} \delta_{u x}{ }^{\prime} \phi_{u x}+\phi_{x u} \delta_{u x}\left(I-\gamma_{x x}\right) \\
& \quad+\left(I-\gamma_{x x}\right)^{\prime} \delta_{u x}{ }^{\prime} \phi_{u u} \delta{ }_{u x}\left(I-\gamma_{x x}\right)
\end{aligned}
$$




$$
\begin{aligned}
& R=\phi_{\mathrm{xu}} \delta_{\mathrm{ux}}+\left(I-\gamma_{\mathrm{xx}}\right)^{\prime} \delta_{\mathrm{ux}}{ }^{\prime} \phi_{\mathrm{uu}} \delta_{\mathrm{ux}} \\
& S=\delta_{\mathrm{ux}}{ }^{\prime} \phi_{\mathrm{uu}} \delta_{\mathrm{ux}} .
\end{aligned}
$$

Then the following is well known.

Fact 2: If $\{x(t+1)\}_{t=\tau}^{\infty}$ is an optimal policy for $P$ then the following conditions must hold:

\section{(Euler Condition)}

$$
\begin{aligned}
& (S-R) E_{t} x(t+2)-\left[Q-\left(R+R^{\prime}\right)+\left(1+B^{-1}\right) S\right] E_{t} x(t+1)+B^{-1}(S-R)^{\prime} E_{t} x(t) \\
& =\ell+\left(B^{-1}-1\right) m+(0-P)^{\prime} E_{t} \xi(t+1)+B^{-1} P^{\prime} E_{t} \xi(t), \\
& \forall t \in\{\tau, \tau+1, \ldots\}
\end{aligned}
$$

(Legendre Condition)

$$
Q-\left(R+R^{\prime}\right)+\left(1+8^{-1}\right) S \text { negative semidefinite }
$$

\section{The New Condition}

Now the stage has been set to state and prove the necessity of the new condition.

Lemma 1: If $\delta(t)=x^{+}(t)-x^{-}(t)$, where $\left\{x^{+}(t+1)\right\}_{t=\tau}^{\infty}$ is an optimal policy for $\mathbf{P}$ and $\left\{\mathrm{x}^{-}(t+1)\right\}_{t=\tau}^{\infty}$ is any feasible policy for $\mathbf{P}$ (i.e., satisfies $\left(2^{\prime}\right)-\left(5^{\prime}\right)$, then the following condition must hold:

$$
\begin{aligned}
\lim _{T \rightarrow \infty} E \sum_{t=\tau}^{T} B^{t-\tau}[ & 2 \delta(t+1)^{\prime}(R-S) \delta(t+2) \\
& \left.+\delta(t+1)^{\prime}\left(Q-R-R^{\prime}+\left(1+B^{-1}\right) S\right) \delta(t+1)\right] \leq 0 .
\end{aligned}
$$


Proof: Since $\left.\mathrm{h}\right|^{\mathrm{t}}$ is quadratic, it follows by Taylor's Theorem that:

$$
\begin{aligned}
& \Delta_{\tau}^{T} \equiv E_{\tau} \sum_{t=\tau}^{T} \beta^{t-\tau}\left(\left.h^{+}\right|^{t}-\left.h^{-}\right|^{t}\right) \\
&=E_{\tau} \sum_{t=\tau}^{T} \beta^{t-\tau}\left\{\left.\nabla_{x} h^{+}\right|^{t}\left[x^{+}(t)-x^{-}(t)\right]+\left.\nabla_{v} h^{+}\right|^{t}\left[v^{+}(t)-v^{-}(t)\right]\right. \\
&-\left.\frac{1}{2}\left[x^{+}(t)-x^{-}(t)\right] \cdot \nabla_{x x} h\right|^{t}\left[x^{+}(t)-x^{-}(t)\right] \\
&-\left.\frac{1}{2}\left[x^{+}(t)-x^{-}(t)\right] \cdot \nabla_{x v^{\prime}} h\right|^{t}\left[v^{+}(t)-v^{-}(t)\right] \\
&-\left.\frac{1}{2}\left[v^{+}(t)-v^{-}(t)\right] \cdot \nabla_{v x} h\right|^{t}\left[x^{+}(t)-x^{-}(t)\right] \\
&\left.-\left.\frac{1}{2}\left[v^{+}(t)-v^{-}(t)\right] \cdot \nabla_{v v^{\prime}} h\right|^{t}\left[v^{+}(t)-v^{-}(t)\right]\right\},
\end{aligned}
$$

where $\left.\nabla_{x} h\right|^{t}$ stands for the gradient of $h$ with respect to $x$ evaluated at $h[\xi(t), x(t), v(t)],\left.\nabla_{x x} h\right|^{t}$ stands for the Hessian of $h$ with respect to $x$ evaluated at $h[\xi(t), x(t), v(t)]$, etc.

$$
\text { Since } v(t)=x(t+1)-x(t) \text { and } \delta(t)=x^{+}(t)-x^{-}(t) \text {, we }
$$

have:

$$
\begin{aligned}
\Delta_{\tau}^{T}=E_{\tau} \sum_{t=\tau}^{T} B^{t-\tau} & \left\{\left[\nabla_{x} h^{+}\left|{ }^{t}-\nabla_{v} h^{+}\right|^{t}\right] \delta(t)+\left.\nabla_{v} h^{+}\right|^{t} \delta(t+1)\right. \\
& -\frac{1}{2} \delta(t) \cdot\left[\left.\nabla_{x x} h\right|^{t}-\left.\nabla_{x v} h\right|^{t}-\left.\nabla_{v x} h\right|^{t}+\left.\nabla_{v v} h\right|^{t}\right] \delta(t) \\
& -\frac{1}{2} \delta(t) \cdot\left[\left.\nabla_{x v} h\right|^{t}-\left.\nabla_{v v} h\right|^{t}\right] \delta(t+1) \\
& \left.-\frac{1}{2} \delta(t+1) \cdot\left|\nabla_{v x} h\right|^{t}-\left.\nabla_{v v} h\right|^{t}\right] \delta(t+1) \\
& \left.-\left.\frac{1}{2} \delta(t+1) \cdot h h_{v v}\right|^{t} \delta(t+1)\right\} .
\end{aligned}
$$


Since both $\left\{\mathrm{x}^{+}(t+1)\right\}_{t=\tau}^{\infty}$ and $\left\{\mathrm{x}^{-}(t+1)\right\}_{t=\tau}^{\infty}$ are assumed to be feasible, (4') implies $\delta(\tau)=0$. Then it follows by a change of time indexes that:

$$
\begin{aligned}
& \Delta_{\tau}^{T}=E_{\tau}\left(\sum _ { t = \tau } ^ { T - 1 } \beta ^ { t + 1 - \tau } \left\{\left[\left.\nabla_{x} h^{+}\right|^{t+1}-\left.\nabla_{v} h^{+}\right|^{t+1}\right] \delta(t+1)\right.\right. \\
& -\frac{1}{2} \delta(t+1) \cdot\left[\left.\nabla_{x x} h\right|^{t+1}-\left.\nabla_{x v} h\right|^{t+1}-\left.\nabla_{v x} h\right|^{t+1}\right. \\
& \left.\left.+\left.\nabla_{v v} h\right|^{t+1}\right] \delta(t+1)-\delta(t+1) \cdot\left[\left.\nabla_{x v} h\right|^{t+1}-\left.\nabla_{v v} h\right|^{t+1}\right] \delta(t+2)\right\} \\
& \left.+\sum_{t=\tau}^{T} \beta^{t-\tau}\left\{\left.\nabla_{v} h^{+}\right|^{t} \delta(t+1)-\left.\frac{1}{2} \delta(t+1) \cdot h_{v v}\right|^{t} \delta(t+1)\right\}\right) \\
& =E_{\tau}\left(\beta \sum_{t=\tau}^{T-1} \beta^{t-\tau}\left[\left.\beta^{-1} \nabla_{v} h^{+}\right|^{t}+\left.\nabla_{x} h^{+}\right|^{t+1}-\left.\nabla_{v} h^{+}\right|^{t+1}\right] \delta(t+1)\right. \\
& +{ }^{t-\tau}\left\{\left.\nabla_{v} h^{+}\right|^{T} \delta(T+1)-\left.\frac{1}{2} \delta(T+1)^{\prime} h_{v v}\right|^{T} \delta(T+1)\right\} \\
& -\frac{1}{2} \beta \sum_{t=\tau}^{T-1} B^{t-\tau}\left\{2 \delta(t+1) \cdot\left[\left.\nabla_{x v} h\right|^{t+1}-\left.\nabla_{v v} h\right|^{t+1}\right] \delta(t+2)\right. \\
& +\delta(t+1) \cdot\left[\left.\nabla_{x x} h\right|^{t+1}-\left.\nabla_{x v} h\right|^{t+1}-\left.\nabla_{v x} h\right|^{t+1}\right. \\
& \left.\left.\left.+\left.\left(1+B^{-1}\right) \nabla_{v v} h\right|^{t+1}\right] \delta(t+1)\right\}\right) \text {. }
\end{aligned}
$$

Using the $\mathbf{P}^{\prime}$ notation:

$$
\begin{aligned}
\Delta_{\tau}^{T}=E_{\tau}\left(B \sum _ { t = \tau } ^ { T } \sum ^ { - 1 } \beta ^ { t - \tau } \left[(S-R) x^{+}(t+2)-\left(Q-R-R^{\prime}+S+B-1+S\right) x^{+}(t+1)\right.\right. \\
+B^{-1}(S-R)^{\prime} x^{+}(t)-\ell-(B-1) m-(0-P)^{\prime} \xi(t+1) \\
\\
\left.-B^{-1} P^{\prime} \xi(t)\right]^{\prime} \delta(t+1)
\end{aligned}
$$




$$
\begin{aligned}
& +B^{T-\tau}\left\{\left[S x^{+}(T+1)+(R-S)^{\prime} x^{+}(T)+m+P^{\prime} \xi(T)\right]^{\prime} \delta(T+1)\right. \\
& \left.-\frac{1}{2} \delta(T+1) \cdot S \delta(T+1)\right\} \\
& -\frac{1}{2} B \sum_{t=\tau}^{T-1} B^{t-\tau}\left\{2 \delta(t+1)^{\prime}(S-R) \delta(t+2)\right. \\
& \left.\left.\left.+\delta(t+1)^{\prime}\left(Q-R-R^{\prime}\right)+S+B^{-1} S\right) \delta(t+1)\right\}\right) .
\end{aligned}
$$

Now, since $\Omega_{t} \subset \Omega_{t+1}, \forall t \in N$, it follows that $E_{\tau}(\cdot)=E_{\tau}\left(E_{t}(\cdot)\right)$, $\forall t \in\{\tau, \tau+1, \ldots\}$ (see, e.g., Billingsley [1986, Theorem 34.4]), and since $\left\{\mathrm{x}^{+}(t+1)\right\}_{t=\tau}^{\infty}$ and $\left\{\mathrm{x}^{-}(t+1)\right\}_{t=\tau}^{\infty}$ are assumed to be $\Omega_{t}$-measurable, it follows by $\left(3^{\prime}\right)$ that $\delta(t+1)$ is $\Omega_{t}$-measurable. Therefore, $E_{t} \delta(t+1)=\delta(t+1)$ and $E_{t}[(\cdot) \delta(t+1)]=\left[E_{t}(\cdot)\right] \delta(t+1)$. (See, e.g., Billingsley [1986, Theorem 34.3].) These facts imply that:

$$
\begin{aligned}
& E_{\tau}\left[(S-R) x^{+}(t+2)-\left(Q-R-R^{\prime}+S+B^{-1} S\right) x^{+}(t+1)+B^{-1}(S-R)^{\prime} x^{+}(t)-\ell\right. \\
& \left.-\left\{\left(B^{-1}-1\right) m-(0-P)^{\prime}\right\}(t+1)-B^{-1} P^{\prime} \xi(t)\right]^{\prime} \delta(t+1) \\
& =E_{\tau}\left\{\left[(S-R) E_{t} x^{+}(t+2)-\left(Q-R-R^{\prime}+S+B^{-1} S\right) E_{t} x^{+}(t+1)\right.\right. \\
& +B^{-1}(S-R)^{\prime} E_{t} x(t)-\ell+\left(B^{-1}-1\right) m-(O-P)^{\prime} E_{t} \xi(t+1) \\
& \left.\left.\quad-B^{-1} P^{\prime} E_{t} \xi(t)\right]^{\prime} \delta(t+1)\right\} .
\end{aligned}
$$

Therefore, since $\left\{x^{+}(t+1)\right\}_{t=\tau}^{\infty}$ is assumed to be an optimal policy for $\mathbf{P}$ it follows by ( 8 ) that the first term in the right hand side of $\left(^{*}\right)$ is zero. Furthermore, since $\left(\{\xi(t)\}_{t=\tau}^{\infty},\left\{x^{+}(t)\right\}_{t=\tau}^{\infty}\right)$, $\left(\{\xi(t)\}_{t=\tau}^{\infty},\left\{x^{-}(t)\right\}_{t=\tau}^{\infty}\right) \varepsilon \mathcal{E}_{\tau}^{\xi} \times E_{\tau}^{x}$, it follows that each and every one of the bilinear and quadratic forms of the second term in the right hand side of $\left(^{*}\right)$ goes to zero as $T \rightarrow \infty$. Therefore, 


$$
\lim _{T \rightarrow \infty} \Delta_{\tau}^{T}=-\frac{1}{2} \beta \lim _{T \rightarrow \infty} E \sum_{\tau} \sum_{t=\tau}^{T-1} \beta^{t-\tau}[2 \delta(t+1) \cdot(S-R) \delta(t+1)
$$

$$
\left.+\delta(t+1)^{\prime}\left(Q-R-R^{\prime}+S+\beta^{-1} S\right) \delta(t+1)\right]
$$

Now, since $E(\cdot)=E\left(E_{t}(\cdot)\right), \forall t \in N$, it follows immediately that if $\left\{x^{+}(t+1)\right\}_{t=\tau}^{\infty}$ is an optimal policy for $P$ and $\left\{x^{-}(t+1)\right\}_{t=\tau}^{\infty}$ is any feasible policy for $P$, it must be that (10) holds.

$$
\text { Q.E.D. }
$$

Then, we have the following.

Theorem: Suppose that $\{\delta(t): t \in N\}, \delta(t)$ being defined as in the preceding lemma, happens to be a covariance stationary process. Then

$$
\begin{aligned}
& E\left[2 \delta(\tau+1)^{\prime}(R-S) \delta(\tau+2)+\delta(\tau+1) \cdot\left(Q-\left(R+R^{\prime}\right)+\left(1+B^{-1}\right) S\right) \delta(\tau+1)\right] \leq 0, \\
& \forall \tau \varepsilon N .
\end{aligned}
$$

Several comments are in order. First (11) is indeed new. In particular it is not and neither is it implied by the other second order condition (i.e., the Legendre Condition). Second, what makes this result useful is that if observed data accord with the optimal policy, then their deviations from their nonstationary components should satisfy (11); for these nonstationary components trivially satisfy all the requirements for a feasible solution (as in the first example below). Or if the maintained hypothesis is that the covariance stationary components of the endogenous variables are generated by the optimal solution of the model and a "zero" feasible solution is meaningful (as in West's inventory model), then again, (11) should be satisfied by the covariance- 
stationary component of the endogenous state variables. Thus, (11) provides for a natural and easily implementable test for the validity of the hypothesis that observed data accord with the optimal solution, without having to make strong curvature restrictions or specify the law of motion of the exogenous state variables. Third, it can be easily verified by tracing the steps of the preceding proof that a similar result obtains when (7a) is not imposed.

Also, when $h$ is twice differentiable but is not necessarily quadratic, and a transversality-like condition holds that will make the second term in the right hand side of (*) go to zero at $\mathrm{T} \rightarrow \infty,(10)$ 's counterpart is:

$$
\begin{aligned}
& \lim _{T \rightarrow \infty} E_{t} \sum_{t=\tau}^{T-1} \beta^{t-\tau} \mid \delta(t+1)\left(\left.\nabla_{x v} h^{\theta}\right|^{t+1}-\left.\nabla_{v v} h^{\theta}\right|^{t+1}\right) \delta(t+2) \\
& +\delta(t+1)^{\prime}\left(\left.\nabla_{x x} h^{\theta}\right|^{t+1}-\left.\nabla_{x v} h^{\theta}\right|^{t+1}\right. \\
& \left.\left.-\left.\nabla_{v x} h^{\theta}\right|^{t+1}+\left.\left(1-\beta^{-1}\right) \nabla_{x v} h^{\theta}\right|^{t+1}\right) \delta(t+1)\right] \leq 0
\end{aligned}
$$

where

$$
\begin{aligned}
& \left.h^{\theta}\right|^{t+1}=h\left[\xi(t+1), x^{\theta}(t+1), v^{\theta}(t+1)\right] \\
& x^{\theta}(t+1)=\theta x^{+}(t+1)+(1-\theta) x^{-}(t+1)
\end{aligned}
$$

and

$$
v^{\theta}(t+1)=\theta v^{+}(t+1)+(1-\theta) v^{-}(t+1)
$$


$\theta \varepsilon(0,1)$. To reduce this condition to something like (11), it must be that $E\left[\left.\nabla_{x v} h^{\theta}\right|^{t+1}-\left.\nabla_{v v} h^{\theta}\right|^{t+1}\right]$ and $E\left[\left.\nabla_{x x} h^{\theta}\right|^{t+1}-\right.$ $\left.\left.\left.\nabla_{x v} h^{\theta}\right|^{t+1} \nabla_{v x} h^{\theta}\right|^{t+1}+\left.\left(1-8^{-1}\right) \nabla_{v v} h_{v}^{\theta}\right|^{t+1}\right]$ are constant matrices. This may be the case after an appropriate transformation (e.g., after multiplying by a positive random variable) if $\left\{x^{\theta}(t)\right\}_{t=\tau}^{\infty}$ is a stationary or steady state and $\left\{x^{-}(t+1)\right\}_{t=\tau}^{\infty}$ is obtained as $(1-\theta)^{-1} x^{\theta}(t+1)-\theta(1-\theta)^{-1} x^{+}(t+1)$ for an appropriately chosen $\theta$. Then, note that $\delta(t+1)=x^{+}(t+1)-x^{-}(t+1)=(1-\theta)^{-1}\left[x^{+}(t+1)-\right.$ $\left.x^{\theta}(t+1)\right]$ implies that (11)'s counterpart is effectively independent of $\theta$. So in order to evaluate the new condition, one needs only the covariance-stationary deviation from the steady state.

\section{Two Examples}

This section presents two examples that illustrate the usefulness of the new condition. That is, how one can construct observable covariance-stationary $\{\delta(t): t \varepsilon \mathbf{N}\}$ processes and thereby check for (11). West's condition will be examined in connection with the second example.

An "Open Loop" Alternative

$$
\begin{aligned}
& \text { Let } \Psi(t)=\xi(t)-E \xi(t) \text {. Suppose that } \\
& \Psi(t+1)=A \Psi(t)+E(t+1)
\end{aligned}
$$

where

(12a) $\quad\{z \varepsilon \notin: \operatorname{det}(I-A z)=0\} \cap\{z \varepsilon \Phi:|z|<1\}=\emptyset$ 
$-13-$

(12b) $\quad \varepsilon(t) \sim \mathcal{N}\left(0, \sum\right) \forall t \in N$

(12c) $\quad E \varepsilon(t)^{\prime} \varepsilon\left(t^{\prime}\right)=0, \forall t \neq t^{\prime}$.

It follows that $\left\{\Psi(t): t_{E N}\right\}$ is a covariance-stationary process.

Assume that:

(bb) $\quad \operatorname{det}(S-R) \neq 0$

and let $\mathrm{J}_{1}\left(\mathrm{~J}_{2}\right)$ be a Jordan matrix with the eigenvalues of:

$$
\varepsilon(z)=I z^{2}-(S-R)^{-1}\left(Q-R-R^{\prime}+S+B^{-1} S\right) z+\beta^{-1}(S-R)^{-1}(S-R)^{\prime}
$$

with modulus less (greater) than $B^{-\frac{1}{2}}$. Also, let $H_{1}\left(H_{2}\right)$ be the matrix with the eigenvectors and the generalized eigenvectors of $\varepsilon(\mathrm{z})$ corresponding to $\mathrm{J}_{1}\left(\mathrm{~J}_{2}\right)$. Then, under the additional regularty restrictions:

(bc) $\quad\{z \varepsilon \notin: \operatorname{det} \varepsilon(z)=0\} \cap\left\{z \varepsilon \notin:|z|=B^{-\frac{1}{2}}\right\}=\emptyset$

(bd) $\quad \operatorname{rank}\left(H_{i}\right)=n_{x}, i=1,2$

the policy for ( 8 ) subject to (4) and (5) is given by: ${ }^{2}, 3$

$$
x^{+}(t+1)=K_{1} x^{+}(t)+M \Psi(t)+N(t), x^{+}(\tau)=\bar{x}
$$

where

$$
\begin{aligned}
& K_{i}=H_{i} J_{i} H_{i}^{-1} \\
& -M=K_{2}^{-1}(S-R)^{-1} B^{-1} P^{\prime}+\sum_{j=1}^{\infty} K_{2}^{-j}\left[(S-R)^{-1}(O-P)^{\prime}+K_{2}^{-1}(S-R)^{-1} B^{-1} P^{\prime}\right] A^{j}
\end{aligned}
$$




$$
\begin{aligned}
-N(t)= & \left(I-K_{2}^{-1}\right)^{-1} K_{2}^{-1}(S-R)^{-1}\left[\ell+\left(B^{-1}-1\right) m\right]+K_{2}^{-1}(S-R)^{-1} B^{-1} P^{\prime} E \xi(t) \\
& +\sum_{j=1}^{\infty} K_{2}^{-j}\left[(S-R)^{-1}(O-P)^{\prime}+K_{2}^{-1}(S-R)^{-1} B^{-1} P^{\prime}\right] E \xi(t+j) .
\end{aligned}
$$

Consider, now, the deterministic problem that results from $P$ by substituting $E \xi(t)$ in (1) and (3) for $\xi(t)$. Under the regularity conditions mentioned earlier, any optimal policy for this problem should satisfy:

$$
x^{-}(t+1)=K_{1} x^{-}(t)+N(t) ; x^{-}(\tau)=\bar{x}
$$

Obviously, $\left\{x^{-}(t+1)\right\}_{t=\tau}^{\infty}$ is a feasible policy for $P$. This policy, sometimes referred to as the "open-loop" policy, and (14) imply:

$$
\begin{aligned}
& x^{+}(t+1)=K_{1}^{t-\tau+1} \bar{x}+\sum_{i=0}^{t-T} K_{1}^{i}[M \Psi(t-i)+N(t-i)] \\
& x^{-}(t+1)=K_{1}^{t-\tau+1} \bar{x}+\sum_{i=0}^{t-T} K_{1}^{i} N(t-i) .
\end{aligned}
$$

Hence,

$$
\delta(t) \equiv x^{+}(+)-x^{-}(+)=\sum_{i=0}^{t-T} K_{1}^{i} M \Psi(t-i) .
$$

Thus, since $\delta(t)$ can be obtained as the finite sum of covariancestationary processes is itself covariance-stationary. It should be observed that this example can be easily extended to account for moving average components in the law of motion of the $\{\psi(t): t \in N\}$ process. 
The "Zero" Alternative

One way of looking at West's inventory model is to consider a firm that takes its sales of a single homogeneous good as given and seeks a production schedule that will minimize its expected discounted future stream of real costs:

$$
E_{0} \sum_{t=0}^{\infty} B^{t}\left\{a_{0}[Q(t)-Q(t-1)]^{2}+a_{1} Q(t)^{2}+a_{2}\left[H(t)-a_{3} S(t+1)\right]^{2}\right\},
$$

$$
a_{i} \varepsilon R, i=0,1,2,3
$$

subject to

$$
Q(t)=S(t)+H(t)-H(t-1)
$$

where $Q(t)$ is production in period $t, H(t)$ is inventories at the end of period $t$, and $s(t+1)$ is the covariance-stationary component of sales in period $t$. The term $a_{0}[Q(t)-Q(t-1)]^{2}$ represents adjustment costs brought about by changing production levels. The term $a_{1} Q(t)^{2}$ represents production costs and the term $a_{2}\left[H(t)-a_{3} S(t+1)\right]^{2}$ represents inventory holding costs and backlog costs. It is hypothesized that the observed covariance-stationary component of production is an optimal policy for this problem. It also follows that $\{Q(t), S(t), H(t): t \in N\}$ form a covariancestationary process.

To map this model in the $P^{\prime}$ format, let:

$$
\begin{gathered}
\xi(t)=(S(t+1) S(t), S(t-1))^{\prime}, x(t)=(H(t-1), H(t-2))^{\prime} \\
N / 2=\left[\begin{array}{ccc}
a_{2} a_{3}^{2} & 0 & 0 \\
0 & a_{0}+a_{1} & -a_{0} \\
0 & 0 & a_{0}
\end{array}\right] \quad 0 / 2=\left[\begin{array}{cc}
-a_{2} a_{3} & 0 \\
-a_{0} & a_{0} \\
a_{0} & -a_{0}
\end{array}\right]
\end{gathered}
$$




$$
\begin{array}{ll}
P / 2=\left[\begin{array}{cc}
-a_{2} a_{3} & 0 \\
a_{0}+a_{1} & 0 \\
-a_{0} & 0
\end{array}\right] & Q / 2=\left[\begin{array}{cc}
a_{0}+a_{2} & -a_{0} \\
-a_{0} & a_{0}
\end{array}\right] . \\
R / 2=\left[\begin{array}{cc}
-a_{0}+a_{2} & 0 \\
a_{0} & 0
\end{array}\right] & S / 2=\left[\begin{array}{cc}
a_{0}+a_{1}+a_{2} & 0 \\
0 & 0
\end{array}\right] .
\end{array}
$$

It can be easily verified that the Euler condition is:

(8')

$$
\begin{aligned}
& {\left[\begin{array}{cc}
2 a_{0}+a_{1} & 0 \\
-a_{0} & 0
\end{array}\right] E_{t}\left[\begin{array}{l}
x_{1}(t+2) \\
x_{2}(t+2)
\end{array}\right]-\left[\begin{array}{cc}
\left(4+\beta^{-1}\right) a_{0}+\left(1-\beta^{-1}\right) a_{1}+\beta^{-1} a_{2} & -2 a_{0} \\
-2 a_{0} & a_{0}
\end{array}\right] E_{t}\left[\begin{array}{l}
x_{1}(t+1) \\
x_{2}(t+1)
\end{array}\right]} \\
& +\beta^{-1}\left[\begin{array}{cc}
2 a_{0}+a_{1} & -a_{0} \\
0 & 0
\end{array}\right] E_{t}\left[\begin{array}{l}
x_{1}(t) \\
x_{2}(t)
\end{array}\right]=\left[\begin{array}{ccc}
0 & -\left(2 a_{0}+a\right) & 2 a_{0} \\
0 & a_{0} & -a_{0}
\end{array}\right] E_{t}\left[\begin{array}{l}
\xi_{1}(t+1) \\
\xi_{2}(t+1) \\
\xi_{3}(t+1)
\end{array}\right] \\
& +B^{-1}\left[\begin{array}{ccc}
-a_{2} a_{3} & a_{0}+a_{1} & -a_{0} \\
0 & 0 & 0
\end{array}\right] E_{t}\left[\begin{array}{l}
\xi_{1}(t) \\
\xi_{2}(t) \\
\xi_{3}(t)
\end{array}\right]=0, \forall t \varepsilon N
\end{aligned}
$$

and the Legendre condition is:

$$
\left[\begin{array}{cr}
\left(4+\beta^{-1}\right) a_{0}+\left(1-\beta^{-1}\right) a_{1}+\beta^{-1} Q_{2} & -2 a_{0} \\
-2 a_{0} & a_{0}
\end{array}\right] \text { positive semidefinite. }
$$

Before looking at (11) it should be helpful and reassuring to verify that $\left(8^{\prime}\right)$ is indeed West's Euler Condition. To do this, first note that the second equation in $\left(8^{\prime}\right)$ is:

$$
a_{0} E_{t}\left[H_{t+1}-H_{t}\right]-a_{0}\left[H_{t}-H_{t-1}\right]+a_{0} E_{t}\left[S_{t+1}-S_{t}\right]=0 .
$$

Then, shift this one period forward keeping the same expectations operator and multiply both sides of the result by $B$. Finally, add the new result to the first line of $\left(8^{\prime}\right)$ and multiply by $B$. This gives West's equation (8). 
Now, the new condition gives:

$$
\begin{gathered}
E\left\{2\left[\begin{array}{l}
\delta_{1}(t+1) \\
\delta_{2}(t+1)
\end{array}\right],\left[\begin{array}{rr}
-\left(2 a_{0}+a_{1}\right) & 0 \\
a_{0} & 0
\end{array}\right]\left[\begin{array}{l}
\delta_{1}(t+1) \\
\delta_{2}(t+1)
\end{array}\right]\right. \\
\left.+\left[\begin{array}{l}
\delta_{1}(t+1) \\
\delta_{2}(t+1)
\end{array}\right]\left[\begin{array}{rr}
\left(4+\beta^{-1} a_{0}+\left(1-\beta^{-1}\right) a_{1}+\beta^{-1} a_{2}\right. & -2 a_{0} \\
-2 a_{0} & a_{0}
\end{array}\right]\left[\begin{array}{l}
\delta_{1}(t+1) \\
\delta_{2}(t+1)
\end{array}\right]\right\} \geq 0
\end{gathered}
$$

or

$\left(11^{\prime}\right)$

$$
\begin{aligned}
& E\left\{\left[\left(5+B^{-1}\right) a_{0}+\left(1+B^{-1}\right) a_{1}+B^{-1} a_{2}\right] H(t)^{2}-2\left(4 a_{0}+a_{1}\right) H(t) H(t-1)\right. \\
& \left.+2 a_{0} H(t) H(t+2)\right\} \geq 0 .
\end{aligned}
$$

But West's corresponding condition (i.e., (6)) is:

$$
\begin{aligned}
& E\left(a_{0}\left\{[S(t)-S(t-1)]^{2}-[Q(t)-Q(t-1)]^{2}\right\}+a_{1}\left\{S(t)^{2}-Q(t)^{2}\right\}\right. \\
& \left.-a_{2} H(t)^{2}+2 a_{2} a_{3} H(t) S(t+1)\right) \geq 0 .
\end{aligned}
$$

The following clarifies the difference between (11') and (16).

Lemma 2: Given $\mathrm{H}^{-}(-2), \mathrm{H}^{-}(-1), \mathrm{H}^{-}(0), \ldots=0,\left(11^{\prime}\right)$ holds if and only if (16) holds.

Proof: Since $\{Q(t), S(t), H(t): t \varepsilon N\}$ is a covariance stationary process, and since $E\left[E_{0}(\cdot)\right]=E(\bullet)$, we have:

$$
\begin{gathered}
\Phi=(1-\beta)^{-1} E\left(a_{0}\left\{[S(t)-S(t-1)]^{2}-[Q(t)-Q(t-1)]^{2}\right\}+a_{1}\left[S(t)^{2}-Q(t)^{2}\right]\right. \\
\left.-a_{2} H(t)^{2}+2 a_{2} a_{3} H(t) S(t+1)\right) \\
=E E_{0} \sum_{t=0}^{\infty} B^{t}\left(a_{0}\left\{[S(t)-S(t-1)]^{2}-[Q(t)-Q(t-1)]^{2}\right\}+a_{1}\left[S(t)^{2}-Q(t)^{2}\right]\right. \\
\left.-a_{2} H(t)^{2}+2 a_{2} a_{3} H(t) S(t+1)\right)
\end{gathered}
$$




$$
\begin{aligned}
& \equiv E_{0} \sum_{t=0}^{\infty} B^{t}\left[\begin{array}{c}
S(t+1) \\
S(t) \\
S(t-1) \\
H(t-1) \\
H(t-2) \\
H(t)-H(t-1) \\
H(t-1)-H(t-2)
\end{array}\right],\left[\begin{array}{ccccccc}
a_{2} a_{3}^{2} & 0 & 0 & -a_{2} a_{3} & 0 & -a_{2} a_{3} & 0 \\
& a_{0}+a_{1} & -a_{0} & -a_{0} & a_{0} & a_{0}+a_{1} & 0 \\
& & a_{0} & -a_{0} & -a_{0} & -a_{0} & 0 \\
& & & a_{0}+a_{2} & -a_{0} & -a_{0}+a_{2} & 0 \\
& & & & a_{0} & a_{0} & 0 \\
& & & & & a_{0}+a_{1}+a_{2} & 0 \\
& & & & & & 0
\end{array}\right]\left[\begin{array}{c}
S(t+1) \\
S(t) \\
S(t-1) \\
H(t-1) \\
H(t-2) \\
H(t)-H(T-1)-H(t-2)
\end{array}\right] \\
& =E E_{0} \sum_{t=0}^{\infty} \beta^{t}\left[\begin{array}{l}
\xi(t) \\
x^{+}(t) \\
v^{+}(t)
\end{array}\right],\left[\begin{array}{lll}
0 & 0 & P \\
O^{\prime} & Q & R \\
P^{\prime} & R^{\prime} & S
\end{array}\right]\left[\begin{array}{l}
\xi(t) \\
x^{+}(t) \\
v^{+}(t)
\end{array}\right] \text {. }
\end{aligned}
$$

On the other hand, it follows as in the Proof of Lemma 1 that:

$$
\begin{aligned}
& X \equiv-\frac{1}{2} B(1-\beta)^{-1} E\left[\left[\left(5+\beta^{-1}\right) a_{0}+\left(1+\beta^{-1}\right) a_{1}+\beta^{-1} a_{2}\right] H(t)^{2}\right. \\
& \left.-2\left(4 a_{0}+a_{1}\right) H(t) H(t-1)+2 a_{0} H(t) H(t-2)\right\} \\
& =E\left(E_{0} \sum_{t=0}^{\infty} \beta^{t}\left\{\left[\begin{array}{l}
\xi(t) \\
x^{+}(t) \\
v^{+}(t)
\end{array}\right],\left[\begin{array}{lll}
N & 0 & P \\
O^{\prime} & Q & R \\
P^{\prime} & R^{\prime} & S
\end{array}\right]\left[\begin{array}{l}
\xi(t) \\
x^{+}(t) \\
v^{+}(t)
\end{array}\right]-\left[\begin{array}{l}
\xi(t) \\
x^{-}(t) \\
v^{-}(t)
\end{array}\right] \cdot\left[\begin{array}{lll}
N & 0 & P \\
O^{\prime} & Q & R \\
P^{\prime} & R^{\prime} & S
\end{array}\right]\left[\begin{array}{l}
\xi(t) \\
x^{-}(t) \\
v^{-}(t)
\end{array}\right]\right\}\right)
\end{aligned}
$$

It follows that if $\mathrm{H}^{-}(-2)=\mathrm{H}^{-}(-1)=\mathrm{H}^{-}(\tau)=\ldots \equiv 0, \Phi=\mathrm{X}$. Hence, since $B \in(0,1)\left(11^{\prime}\right)$ holds if and only if (16) holds.

$$
\text { Q.E.D. }
$$

This resuit also makes clear the way (16) was derived; that is, without using the other necessary conditions. Although, for this model the difference between (11) and (16) is rather unimportant, it should be obvious that for models where some exogenous state variables are not observable (i.e., preference and technology shocks) or when a "zero" alternative feasible solution does not exist this difference is crucial." 


\section{Footnotes}

${ }^{1}$ For examples of LRE models see Hansen and Sargent [1981] and Sargent [1982, 1985].

${ }^{2}$ See Kollintzas [1986a, 1986b].

${ }^{3}$ The regularity conditions $(6 b),(6 c)$, and $(6 d)$ are discussed in Kollintzas [1985, 1986b]. Condition (6c) is somewhat stronger than necessary. These conditions are not sufficient for (13) to be a solution to $P$.

"For example, this is true for a variety of LRE inventory models. See, e.g., Kollintzas and Husted [1984]. 
References

Billingsley, P. (1986). Probability and Measure, New York: Wiley.

Hansen, L. and Sargent, T. (1981). "Linear Rational Expectations Models for Dynamically Interrelated Variables," $\mathrm{R}$. Lucas and T. Sargent, eds., Rational Expectations and Econometric Practice, Minneapolis: University of Minnesota Press. Kollintzas, T. (1985). "The Symetric Linear Rational Expectations Model," Econometrica 53, 963-76.

- (1986a). "A Non-Recursive Solution for the

Linear Rational Expectations Model," Journal of Economic Dynamics and Control $10,327-32$.

- (1986b). "Some New Results Concerning the

Solution to Linear Rational Expectations Models Arising From Optimizing Behavior," Working Paper No. 204, Department of Economics, University of Pittsburgh. and Husted, S. (1984). "Distributed Lags and

Intermediate Good Imports," Journal of Economic Dynamics and Control 8, 303-27.

Sargent, T. (1982). "Lecture Notes on Linear Optimal Control, Filtering, and Rational Expectations," Working Paper No. 224, Research Department, Federal Reserve Bank of Minneapolis. , ed. (1985). Energy, Eoresight and Strategy, Washington, D.C.: Resources for the Future. West, K. (1986). "A Variance Bounds Test of the Linear Quadratic Inventory Problem," Journal of Political Economy 94, 374-401. 